\title{
Effects of Low Light Stress on Growth Parameters of Rice (Oryza sativa L.) Genotypes
}

\author{
Aradhana Dhruw* and Arti Guhey \\ Department of Plant Physiology, College of agriculture Raipur (IGKV, RAIPUR), India \\ *Corresponding author
}

A B S T R A C T

\section{Keywords}

Low light stress, Growth parameters, Oryza sativa, Genotypes

Article Info

Accepted:

06 June 2018

Available Online:

10 July 2018
Light is a major constraint for the rice production. Twenty five rice genotypes was exposed to normal light and low light to study some growth parameters. The statistical analysis showed significant difference between the normal light and low light condition. Under low light condition the growth parameters specific leaf weight (SLW), Relative growth rate (RGR), net assimilation rate (NAR), crop growth rate (CGR) were decreased whereas specific leaf area (SLA), leaf area ratio (LAR), leaf area index (LAI) were increased. The genotype DXD (124)-17-192 and DXD (124)-17-193 exhibited higher specific leaf weight (SLW) and net assimilation rate (NAR).

\section{Introduction}

Rice (Oryza sativa L.) is the world single most important food crop, being the primary source for more than one third of the world population. Solar radiation is a limited during the monsoon season and in July and August, the average daily duration of bright sunshine in only 2-3 hours per day. Lower incidence of solar radiation coupled with fluctuating light intensity due to overcast sky sun during the wet season (Feb. to Nov.) is one of the major constraints for rice production in eastern India. Light intensity is a basic characteristic asset, which controls morphogenesis and production in plants. It has a major role in photosynthesis, photoperiodism, and photonasty. In fact, agriculture can be defined as the exploitation of solar radiation with the help of water and nutrients (Fageria, 2013). The Light intensity is among essential prerequisites for plant development, advancement, survival, and yield efficiency (Wang, 2013). Under low light during the grain-filling stage decreased supply of carbohydrates to grains as well as a decrease in starch synthase activity in grains, which directly inhibits grain filling and enhances the occurrence of chalky rice (Li et al., 2005). 


\section{Materials and Methods}

The field experiment was laid out in split plot design and consisted of 160 rice genotype (139 RILs lines and 17 released varieties from IGKV, Raipur and 4 varieties from NRRI) under normal condition and shade net (70:30) condition (low light condition) and net shade condition had provided at 40 days after transplanting whereas, grown 25 selected from 160 rice genotype and light intensity was measured by lux meter at different growth stages under both condition (normal light and low light condition).

\section{Results and Discussion}

Significant differences were noticed among the treatment in relation to specific leaf area (Table 1 and Fig. 1). Specific leaf area $\left(\mathrm{cm}^{2}\right.$ $/ g)$ at $50 \%$ flowering stage of crop was increased under low light as compare to normal light. Overall mean increase in specific leaf area was noticed $44 \%$ under low light condition and variety DXD (124)-17-193 attained minimum specific leaf area under low light stress. Lower SLA was seen in rice genotype grown in a normal light condition which is in consistence with the findings of these authors. For shaded rice genotype, the increased SLA and the development of a darker green colour were mainly attributed to the higher nitrogen content found in their leaves. It is likely that the increased SLA and the development of dark green leaf colour under shaded rice genotype partly contributed for the higher rate of photosynthesis observed under this condition. Similar result was also noticed by Robakowski et al., (2003).

The data on specific leaf weight presented in Table 1 and Figure 2. Specific leaf weight (g/ $\mathrm{cm}^{2}$ ) at $50 \%$ flowering stage of crop was decreased under low light as compare to normal light condition by $30 \%$. Rice genotype DXD (124)-17-192 exhibited maximum specific leaf weight (SLW) and showed significant and positive association with positive and significant correlation with grain yield. Under low light condition SLW was significantly decreased as compare to normal light condition. (Koumoto et al., 2016) Shade decreased the specific leaf weight and the leaf sugar and starch contents. Low light condition during vegetative growth increased the susceptibility of rice plants to chilling damage during panicle development.

Relative growth rate $(\mathrm{mg} / \mathrm{g} /$ day) at vegetative stage of crop was decreased under low light condition as compare to normal light (Table 2 and Fig. 3). Overall mean reduce in relative growth rate was noticed $40 \%$ under low light condition and showed positive and significant correlation with net assimilation rate, grain yield and harvest index (Table 3 ).

The rice genotype DXD (124)-17-193 exhibited maximum relative growth rate (RGR).In low light condition RGR was significantly reduced. Similar result was found by Janardhan et al., (1980).

Net assimilation rate $\left(\mathrm{mg} / \mathrm{dm}^{2}\right.$ leaf area/day) at vegetative stage of crop was decreased under low light condition as contrast with normal light Table 2 and Fig. 4). Rice genotype DXD (124)-17-192 exhibited maximum NAR. It was reduced 20\% under low light stress and showed significant and positive association with crop growth rate and grain yield under low light intensity. Ahmad et al., (2009) reported that the low light can affect the morphological, physiological and yield parameters of rice crops such as the source sink relationships, LAI, crop growth rate (CGR), net assimilation rate (NAR), number of panicles $\mathrm{m}^{-2}$. Janardhan and Murty (1980) also found that the dry matter production under low light impaired through reduced photosynthetic rate and net assimilation rate in rice. 
Table.1 Effect of normal light and low light condition on specific leaf area (SLA) and specific leaf weight (SLW) at 50\% flowering in rice genotypes

\begin{tabular}{|c|c|c|c|c|c|c|c|c|c|c|c|c|c|c|c|c|c|c|c|}
\hline \multirow{3}{*}{ S.N. } & \multirow{3}{*}{ Name of entries } & \multicolumn{6}{|c|}{$\mathrm{FLA}\left(\mathrm{cm}^{-2}\right)$} & \multicolumn{6}{|c|}{$\operatorname{SLA}\left(\mathrm{cm}^{2} \mathrm{~g}^{-1}\right)$} & \multicolumn{6}{|c|}{$\operatorname{SLW}\left(\mathrm{g} \mathrm{cm}^{2}\right)$} \\
\hline & & \multicolumn{2}{|c|}{2015} & \multicolumn{2}{|c|}{2016} & \multicolumn{2}{|c|}{ Pooled } & \multicolumn{2}{|c|}{2015} & \multicolumn{2}{|c|}{2016} & \multicolumn{2}{|c|}{ Pooled } & \multicolumn{2}{|c|}{2015} & \multicolumn{2}{|c|}{2016} & \multicolumn{2}{|c|}{ Pooled } \\
\hline & & NL & $\mathbf{L L}$ & NL & $\mathbf{L L}$ & NL & $\mathbf{L L}$ & NL & $\mathbf{L L}$ & NL & $\mathbf{L L}$ & NL & $\mathbf{L L}$ & NL & $\mathbf{L L}$ & NL & $\mathbf{L L}$ & NL & $\mathbf{L L}$ \\
\hline 1 & DXD(124)-1-10 & 62.8 & 65.0 & 59.4 & 63.9 & 61.1 & 64.4 & 318.1 & 453.9 & 282.5 & 354.5 & 300.3 & 404.2 & 0.0045 & 0.0027 & 0.0073 & 0.0056 & 0.0059 & 0.0041 \\
\hline 2 & $\mathrm{DXD}(124)-1-12$ & 67.7 & 81.5 & 78.6 & 88.6 & 73.2 & 85.1 & 264.5 & 447.3 & 239.1 & 348.7 & 251.8 & 398.0 & 0.0049 & 0.0026 & 0.0076 & 0.0057 & 0.0062 & 0.0042 \\
\hline 3 & $\mathrm{DXD}(124)-2-22$ & 38.6 & 45.5 & 40.0 & 41.0 & 39.3 & 43.3 & 253.8 & 432.5 & 229.7 & 336.4 & 241.8 & 384.4 & 0.0045 & 0.0035 & 0.0091 & 0.0065 & 0.0068 & 0.0050 \\
\hline 4 & DXD(124)-3-28 & 31.7 & 41.9 & 34.6 & 40.6 & 33.2 & 41.3 & 225.9 & 427.0 & 269.5 & 354.5 & 247.7 & 390.8 & 0.0086 & 0.0042 & 0.0087 & 0.0063 & 0.0087 & 0.0053 \\
\hline 5 & $\mathrm{DXD}(124)-5-72$ & 22.2 & 25.9 & 25.1 & 33.6 & 25.2 & 29.4 & 387.7 & 474.1 & 266.4 & 399.5 & 327.0 & 436.8 & 0.0052 & 0.0037 & 0.0067 & 0.0040 & 0.0060 & 0.0038 \\
\hline 6 & DXD(124)-9-89 & 31.9 & 34.1 & 33.0 & 37.6 & 32.5 & 35.8 & 281.7 & 325.9 & 208.5 & 333.9 & 245.1 & 329.9 & 0.0067 & 0.0063 & 0.0070 & 0.0055 & 0.0068 & 0.0060 \\
\hline 7 & DXD(124)-9-91 & 33.7 & 38.1 & 35.9 & 48.8 & 34.8 & 43.4 & 224.4 & 426.9 & 275.3 & 364.3 & 249.9 & 395.6 & 0.0087 & 0.0054 & 0.0112 & 0.0076 & 0.0099 & 0.0065 \\
\hline 8 & DXD(124)-11-133 & 45.9 & 42.5 & 46.7 & 50.6 & 46.3 & 46.6 & 469.1 & 524.8 & 263.8 & 378.5 & 366.4 & 451.7 & 0.0039 & 0.0035 & 0.0086 & 0.0067 & 0.0063 & 0.0051 \\
\hline 9 & DXD(124)-13-148 & 29.4 & 52.6 & 35.1 & 44.6 & 32.3 & 48.6 & 323.8 & 485.0 & 323.8 & 420.0 & 323.8 & 452.5 & 0.0063 & 0.0036 & 0.0081 & 0.0029 & 0.0072 & 0.0033 \\
\hline 10 & DXD(124)-15-164 & 44.7 & 38.1 & 34.2 & 41.4 & 39.4 & 39.7 & 262.5 & 355.4 & 332.8 & 490.5 & 297.6 & 422.9 & 0.0099 & 0.0067 & 0.0063 & 0.0051 & 0.0081 & 0.0059 \\
\hline 11 & DXD(124)-15-168 & 36.9 & 52.9 & 47.3 & 66.5 & 42.1 & 59.7 & 272.7 & 403.2 & 236.9 & 341.9 & 254.8 & 372.6 & 0.0074 & 0.0051 & 0.0081 & 0.0043 & 0.0078 & 0.0047 \\
\hline 12 & DXD(124)-16-179 & 24.9 & 36.0 & 29.5 & 30.4 & 27.2 & 33.2 & 271.7 & 434.7 & 440.5 & 544.8 & 356.1 & 489.7 & 0.0074 & 0.0046 & 0.0072 & 0.0037 & 0.0073 & 0.0041 \\
\hline 15 & DXD(124)-17-191 & 47.1 & 47.2 & 45.4 & 47.2 & 46.2 & 47.2 & 431.3 & 438.3 & 452.9 & 553.9 & 442.1 & 496.1 & 0.0046 & 0.0038 & 0.0098 & 0.0079 & 0.0072 & 0.0058 \\
\hline 14 & DXD(124)-17-192 & 36.3 & 43.2 & 39.4 & 38.4 & 37.9 & 40.8 & 341.3 & 481.4 & 201.0 & 353.7 & 271.1 & 417.6 & 0.0065 & 0.0054 & 0.0102 & 0.0054 & 0.0077 & 0.0068 \\
\hline 15 & DXD(124)-17-193 & 28.6 & 54.2 & 35.6 & 43.6 & 32.1 & 48.9 & 305.6 & 325.5 & 242.4 & 344.5 & 274.0 & 335.0 & 0.0056 & 0.0036 & 0.0069 & 0.0048 & 0.0079 & 0.0045 \\
\hline 16 & DXD(124)-17-210 & 29.2 & 44.5 & 32.5 & 36.9 & 30.9 & 40.7 & 336.7 & 469.6 & 298.0 & 395.3 & 317.3 & 432.5 & 0.0091 & 0.0054 & 0.0089 & 0.0082 & 0.0080 & 0.0051 \\
\hline 17 & DXD(124)-21-262 & 26.0 & 30.2 & 28.1 & 33.7 & 27.1 & 31.9 & 290.7 & 352.3 & 318.0 & 469.3 & 304.3 & 410.8 & 0.0069 & 0.0054 & 0.0073 & 0.0054 & 0.0071 & 0.0054 \\
\hline 15 & SWARNA SUB 1 & 38.3 & 47.3 & 40.8 & 45.9 & 39.5 & 46.6 & 429.4 & 552.0 & 431.3 & 702.2 & 430.3 & 427.1 & 0.0065 & 0.0050 & 0.0064 & 0.0049 & 0.0064 & 0.0049 \\
\hline 19 & SAMLESHWARI & 54.3 & 57.9 & 55.3 & 62.7 & 54.8 & 60.3 & 238.8 & 314.3 & 246.3 & 361.2 & 242.5 & 337.7 & 0.0094 & 0.0064 & 0.0086 & 0.0051 & 0.0090 & 0.0057 \\
\hline 20 & IBD-1 & 42.6 & 46.6 & 44.9 & 55.6 & 43.7 & 51.1 & 265.7 & 352.9 & 196.9 & 377.7 & 231.3 & 365.3 & 0.0075 & 0.0051 & 0.0091 & 0.0076 & 0.0083 & 0.0063 \\
\hline 21 & IGKV R-1(RAJESHWARI) & 34.7 & 34.8 & 34.7 & 44.8 & 34.7 & 39.8 & 359.9 & 372.3 & 233.6 & 323.7 & 296.7 & 348.0 & 0.0075 & 0.0057 & 0.0089 & 0.0074 & 0.0082 & 0.0066 \\
\hline 22 & IGKV R-2(MAHESHWARI) & 29.6 & 29.2 & 29.4 & 41.4 & 29.5 & 35.3 & 337.9 & 433.4 & 287.7 & 476.2 & 312.8 & 454.8 & 0.0059 & 0.0042 & 0.0086 & 0.0041 & 0.0072 & 0.0041 \\
\hline 23 & JALDUBI & 81.4 & 85.4 & 82.7 & 92.9 & 82.1 & 89.1 & 281.8 & 437.2 & 250.5 & 351.8 & 266.1 & 394.5 & 0.0071 & 0.0049 & 0.0088 & 0.0080 & 0.0079 & 0.0064 \\
\hline 24 & ISD-1 & 33.8 & 33.8 & 33.8 & 39.2 & 33.8 & 36.5 & 317.4 & 456.2 & 244.8 & 324.4 & 281.1 & 390.3 & 0.0063 & 0.0044 & 0.0108 & 0.0063 & 0.0086 & 0.0054 \\
\hline \multirow[t]{7}{*}{25} & INDIRA SONA & 53.8 & 58.7 & 33.7 & 44.2 & 43.8 & 51.4 & 371.1 & 463.7 & 338.4 & 532.9 & 354.8 & 498.3 & 0.0048 & 0.0037 & 0.0085 & 0.0078 & 0.0067 & 0.0057 \\
\hline & TRATMENT MEAN & 40.4 & 46.6 & 41.4 & 48.6 & 40.9 & 47.6 & 314.5 & 425.6 & 284.4 & 409.4 & 299.5 & 417.5 & 0.0066 & 0.0046 & 0.0083 & 0.0058 & 0.0074 & 0.0052 \\
\hline & & CD & SEM & CD & SEM & CD & SEM & CD & SEM & CD & SEM & CD & SEM & CD & SEM & CD & SEM & CD & SEM \\
\hline & Treatment(T) & N/A & 0.374 & 1.67 & 0.093 & 4.289 & 0.239 & 51.76 & 2.88 & 56.98 & 3.171 & 27.06 & 1.506 & 0.0008 & 0.0001 & 0.0011 & 0.00006 & 0.0001 & 0.00165 \\
\hline & Varieties(V) & 4.86 & 1.705 & 4.38 & 1.536 & 3.033 & 1.063 & 29.79 & 10.44 & 41.19 & 14.44 & 27.205 & 9.538 & 0.0005 & 0.0001 & 0.0003 & 0.00014 & 0.0003 & 0.00011 \\
\hline & V X T & 7.77 & 1.869 & 6.26 & 0.465 & 4.87 & 1.194 & 50.63 & 14.40 & 65.81 & 15.85 & 41.087 & 7.53 & 0.0007 & 0.0002 & 0.0005 & 0.00020 & 0.0004 & 0.00016 \\
\hline & T X V & 7.71 & 2.393 & 6.14 & 2.131 & 4.834 & 1.492 & 50.58 & 14.75 & 65.29 & 20.26 & 40.518 & 13.30 & 0.0003 & 0.0002 & 0.0001 & 0.00020 & 0.0004 & 0.00016 \\
\hline
\end{tabular}

$\mathrm{NL}=$ normal light, $\mathrm{LL}=$ low light, $\mathrm{N} / \mathrm{A}=$ non-significant, $\mathrm{CD}=$ critical difference, $\mathrm{SEM}=$ standard error of mean. 
Table.2 Effect of normal light and low light condition on relative growth rate (RGR), net assimilation rate (NAR) and crop growth rate (CGR) at vegetative stage in rice genotype

\begin{tabular}{|c|c|c|c|c|c|c|c|c|c|c|c|c|c|c|c|c|c|c|c|}
\hline \multirow{3}{*}{ S.N. } & \multirow{3}{*}{ Name of genotype } & \multicolumn{6}{|c|}{$\operatorname{RGR}\left(\mathrm{mg} \mathrm{g}^{-1} \mathrm{day}^{-1}\right)$} & \multicolumn{6}{|c|}{ NAR $\left(\mathrm{mg} \mathrm{dm}^{-2}\right.$ leaf area day $\left.{ }^{-1}\right)$} & \multicolumn{6}{|c|}{$\operatorname{CGR}\left(\mathrm{g} \mathrm{m}_{-}^{-2}\right.$ day- $\left.^{-1}\right)$} \\
\hline & & \multicolumn{2}{|l|}{2015} & \multicolumn{2}{|c|}{2016} & \multicolumn{2}{|l|}{ Pooled } & \multicolumn{2}{|l|}{2015} & \multicolumn{2}{|c|}{2016} & \multicolumn{2}{|l|}{ Pooled } & \multicolumn{2}{|l|}{2015} & \multicolumn{2}{|c|}{2016} & \multicolumn{2}{|l|}{ Pooled } \\
\hline & & NL & LL & NL & $\mathbf{L L}$ & NL & $\mathbf{L L}$ & NL & LL & NL & LL & & LL & NL & LL & NL & LL & & $\mathbf{L L}$ \\
\hline 1 & DXD(124)-1-10 & 0.627 & 0.497 & 0.594 & 0.364 & 0.611 & 0.431 & 0.0080 & 0.0050 & 0.0070 & 0.0030 & 0.0060 & 0.0030 & 2.555 & 2.085 & 1.160 & 1.010 & 1.858 & 1.548 \\
\hline 2 & DXD(124)-1-12 & 0.457 & 0.344 & 0.751 & 0.105 & 0.604 & 0.225 & 0.0040 & 0.0030 & 0.0110 & 0.0020 & 0.0060 & 0.0020 & 1.795 & 1.220 & 1.105 & 1.035 & 1.450 & 1.128 \\
\hline 3 & DXD(124)-2-22 & 0.446 & 0.441 & 0.430 & 0.086 & 0.438 & 0.264 & 0.0050 & 0.0040 & 0.0080 & 0.0020 & 0.0040 & 0.0020 & 1.905 & 1.555 & 1.085 & 1.020 & 1.495 & 1.288 \\
\hline 4 & $\mathrm{DXD}(124)-3-28$ & 0.532 & 0.278 & 0.505 & 0.291 & 0.519 & 0.285 & 0.0070 & 0.0040 & 0.0100 & 0.0050 & 0.0060 & 0.0030 & 1.715 & 0.985 & 1.060 & 1.020 & 1.388 & 1.003 \\
\hline 5 & DXD(124)-5-72 & 0.822 & 0.468 & 0.674 & 0.246 & 0.748 & 0.357 & 0.0070 & 0.0060 & 0.0080 & 0.0020 & 0.0050 & 0.0030 & 2.305 & 0.260 & 1.245 & 1.025 & 1.775 & 0.643 \\
\hline 6 & $\mathrm{DXD}(124)-9-89$ & 0.546 & 0.328 & 0.456 & 0.301 & 0.501 & 0.315 & 0.0070 & 0.0070 & 0.0110 & 0.0030 & 0.0070 & 0.0040 & 1.440 & 0.580 & 1.130 & 1.025 & 1.285 & 0.803 \\
\hline 7 & DXD(124)-9-91 & 0.592 & 0.257 & 0.508 & 0.241 & 0.550 & 0.249 & 0.0050 & 0.0030 & 0.0070 & 0.0040 & 0.0040 & 0.0020 & 2.275 & 0.675 & 1.140 & 1.015 & 1.708 & 0.845 \\
\hline 8 & DXD(124)-11-133 & 0.645 & 0.684 & 0.517 & 0.377 & 0.581 & 0.531 & 0.0080 & 0.0070 & 0.0140 & 0.0060 & 0.0090 & 0.0040 & 2.250 & 1.995 & 1.375 & 1.025 & 1.813 & 1.510 \\
\hline 9 & DXD(124)-13-148 & 0.380 & 0.209 & 0.515 & 0.051 & 0.448 & 0.130 & 0.0050 & 0.0030 & 0.0070 & 0.0030 & 0.0040 & 0.0020 & 1.355 & 0.595 & 1.050 & 1.010 & 1.203 & 0.803 \\
\hline 10 & DXD(124)-15-164 & 0.662 & 0.445 & 0.718 & 0.370 & 0.690 & 0.408 & 0.0070 & 0.0050 & 0.0080 & 0.0030 & 0.0060 & 0.0030 & 2.030 & 1.520 & 1.270 & 1.025 & 1.650 & 1.273 \\
\hline 11 & DXD(124)-15-168 & 0.832 & 0.316 & 0.481 & 0.297 & 0.657 & 0.307 & 0.0150 & 0.0090 & 0.0080 & 0.0060 & 0.0090 & 0.0050 & 1.340 & 0.900 & 1.095 & 1.020 & 1.218 & 0.960 \\
\hline 12 & DXD(124)-16-179 & 1.007 & 0.401 & 0.599 & 0.396 & 0.803 & 0.399 & 0.0100 & 0.0060 & 0.0080 & 0.0050 & 0.0070 & 0.0030 & 3.435 & 0.985 & 2.095 & 1.020 & 2.765 & 1.003 \\
\hline 13 & DXD(124)-17-191 & 1.008 & 0.346 & 0.500 & 0.353 & 0.754 & 0.350 & 0.0040 & 0.0050 & 0.0090 & 0.0050 & 0.0050 & 0.0020 & 3.440 & 1.905 & 1.400 & 1.025 & 2.420 & 1.465 \\
\hline 14 & DXD(124)-17-192 & 0.741 & 0.622 & 0.432 & 0.405 & 0.587 & 0.514 & 0.0220 & 0.0170 & 0.0150 & 0.0080 & 0.0160 & 0.0070 & 3.490 & 1.960 & 1.405 & 1.020 & 2.448 & 1.490 \\
\hline 15 & DXD(124)-17-193 & 1.008 & 0.499 & 0.480 & 0.435 & 0.744 & 0.467 & 0.0100 & 0.0070 & 0.0110 & 0.0070 & 0.0090 & 0.0040 & 3.545 & 2.005 & 1.435 & 1.015 & 2.490 & 1.510 \\
\hline 16 & $\operatorname{DXD}(124)-17-210$ & 0.531 & 0.270 & 0.394 & 0.269 & 0.463 & 0.270 & 0.0060 & 0.0040 & 0.0060 & 0.0050 & 0.0060 & 0.0045 & 2.130 & 0.840 & 1.770 & 1.010 & 1.950 & 0.925 \\
\hline 17 & DXD(124)-21-262 & 0.750 & 0.169 & 0.470 & 0.338 & 0.610 & 0.254 & 0.0070 & 0.0030 & 0.0130 & 0.0080 & 0.0080 & 0.0050 & 2.485 & 0.395 & 1.590 & 1.035 & 2.038 & 0.715 \\
\hline 18 & SWARNA SUB 1 & 1.057 & 0.875 & 0.548 & 0.309 & 0.803 & 0.592 & 0.0070 & 0.0050 & 0.0090 & 0.0040 & 0.0060 & 0.0030 & 3.535 & 3.055 & 1.410 & 1.015 & 2.473 & 2.035 \\
\hline 19 & SAMLESHWARI & 0.750 & 0.330 & 0.533 & 0.407 & 0.642 & 0.369 & 0.0110 & 0.0050 & 0.0150 & 0.0100 & 0.0110 & 0.0040 & 2.330 & 1.310 & 1.405 & 1.035 & 1.868 & 1.173 \\
\hline 20 & IBD-1 & 0.638 & 0.181 & 0.882 & 0.574 & 0.760 & 0.378 & 0.0090 & 0.0040 & 0.0080 & 0.0050 & 0.0060 & 0.0020 & 2.065 & 0.660 & 1.060 & 1.025 & 1.563 & 0.843 \\
\hline 21 & IGKV R-1(RAJESHWARI) & 0.557 & 0.519 & 0.929 & 0.760 & 0.743 & 0.640 & 0.0080 & 0.0070 & 0.0090 & 0.0060 & 0.0070 & 0.0050 & 1.980 & 1.785 & 1.260 & 1.050 & 1.620 & 1.418 \\
\hline 22 & IGKV R-2(MAHESHWARI) & 0.745 & 0.700 & 0.669 & 0.457 & 0.707 & 0.579 & 0.0080 & 0.0070 & 0.0100 & 0.0080 & 0.0070 & 0.0030 & 2.735 & 2.440 & 1.395 & 1.030 & 2.065 & 1.735 \\
\hline 23 & JALDUBI & 0.730 & 0.604 & 0.821 & 0.524 & 0.776 & 0.564 & 0.0180 & 0.0110 & 0.0140 & 0.0090 & 0.0140 & 0.0070 & 3.280 & 1.810 & 1.325 & 1.060 & 2.303 & 1.435 \\
\hline 24 & ISD-1 & 0.628 & 0.371 & 0.713 & 0.326 & 0.671 & 0.349 & 0.0050 & 0.0030 & 0.0160 & 0.0030 & 0.0070 & 0.0020 & 0.765 & 0.620 & 1.575 & 1.020 & 1.170 & 0.820 \\
\hline \multirow[t]{7}{*}{25} & INDIRA SONA & 0.839 & 0.659 & 0.357 & 0.015 & 0.598 & 0.337 & 0.0090 & 0.0060 & 0.0070 & 0.0030 & 0.0060 & 0.0020 & 2.950 & 2.440 & 1.050 & 1.020 & 2.000 & 1.730 \\
\hline & TRATMENT MEAN & 0.701 & 0.433 & 0.579 & 0.332 & 0.640 & 0.382 & 0.0085 & 0.0058 & 0.0108 & 0.0054 & 0.0076 & 0.0061 & 2.365 & 1.383 & 1.316 & 1.024 & 1.840 & 1.204 \\
\hline & & CD & SEM & CD & SEM & CD & SEM & CD & SEM & CD & SEM & CD & SEM & CD & SEM & CD & SEM & CD & SEM \\
\hline & Treatment(T) & 0.033 & 0.002 & 0.073 & 0.004 & 0.054 & 0.003 & 0.003 & 0.000 & 0.003 & 0.000 & 0.003 & 0.000 & 0.167 & 0.009 & 0.056 & 0.003 & 0.044 & 0.002 \\
\hline & Varieties(V) & 0.171 & 0.06 & 0.141 & 0.049 & 0.105 & 0.037 & 0.002 & 0.001 & 0.002 & 0.001 & 0.001 & 0.000 & 0.571 & 0.200 & 0.116 & 0.041 & 0.116 & 0.041 \\
\hline & $\mathrm{V} \mathrm{X} \mathrm{T}$ & 0.243 & 0.009 & 0.203 & 0.02 & 0.151 & 0.015 & 0.003 & 0.001 & 0.003 & 0.001 & 0.003 & 0.001 & 0.812 & 0.047 & 0.166 & 0.016 & 0.166 & 0.012 \\
\hline & $\mathrm{TXV}$ & 0.238 & 0.083 & 0.2 & 0.069 & 0.148 & 0.051 & 0.003 & 0.001 & 0.003 & 0.001 & 0.003 & 0.001 & 0.796 & 0.277 & 0.163 & 0.056 & 0.162 & 0.056 \\
\hline
\end{tabular}

$\mathrm{NL}=$ normal light, $\mathrm{LL}=$ low light, $\mathrm{N} / \mathrm{A}=$ non-significant, $\mathrm{CD}=$ critical difference, $\mathrm{SEM}=$ standard error of mean 
Table.3 Correlation analysis among the growth and yield of rice genotype under low light condition

\begin{tabular}{|l|l|l|l|l|l|l|}
\hline & SLA & SLW & RGR & NAR & CGR & GY \\
\hline SLA & 1.000 & 0.056 & -0.127 & 0.238 & 0.109 & -0.063 \\
\hline SLW & & 1.000 & 0.111 & 0.365 & 0.341 & $0.408^{*}$ \\
\hline RGR & & & 1.000 & $0.455^{*}$ & 0.217 & $0.404^{*}$ \\
\hline NAR & & & & 1.000 & $0.539^{* *}$ & $0.426^{*}$ \\
\hline CGR & & & & & 1.000 & 0.017 \\
\hline GY & & & & & & 1.000 \\
\hline
\end{tabular}

Significance at the $5 \%$ and $1 \%$ levels are indicated by $*, * *$ respectively. SLA=Specific leaf area, SLW=Specific leaf weight, $\mathrm{RGR}=$ Relative growth rate, $\mathrm{NAR}=\mathrm{Net}$ assimilation rate, $\mathrm{CGR}=\mathrm{Crop}$ growth rate and $\mathrm{GY}=$ Grain yield.

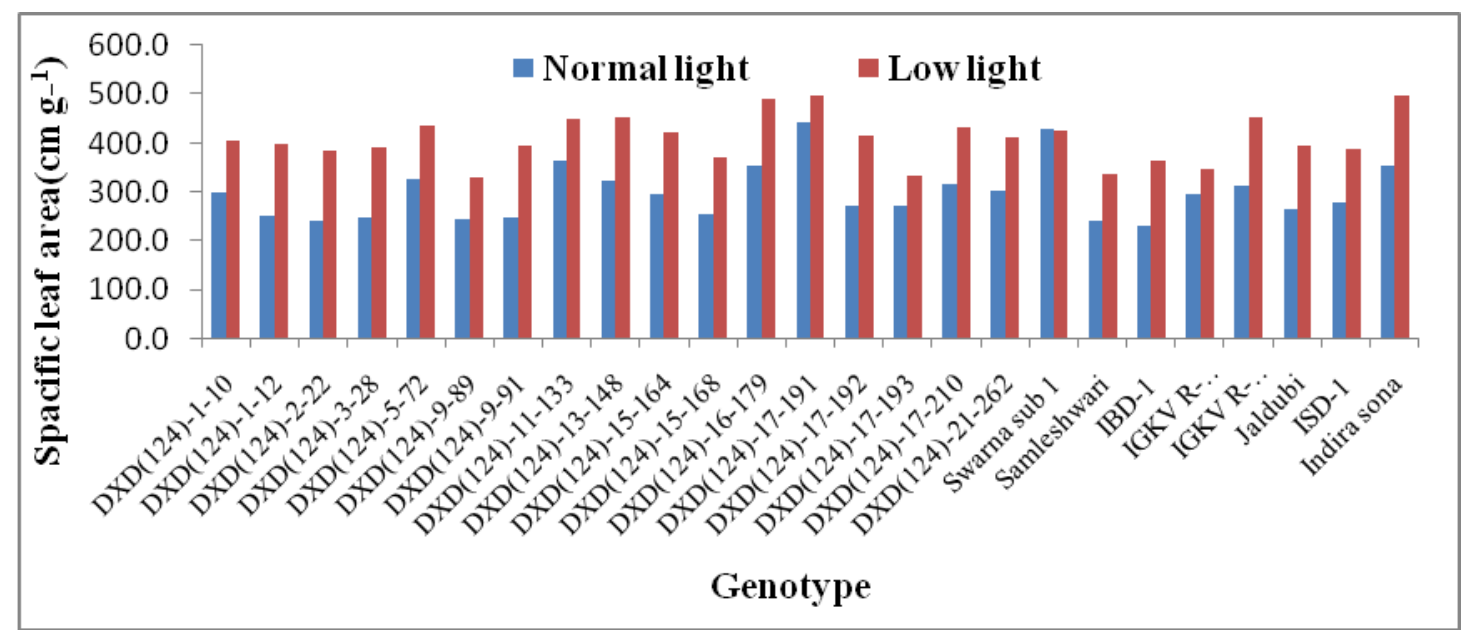

Fig.1 Effects of normal light (NL) and low light (LL) conditions on specific leaf area of rice (Oryza sativa L.) genotypes

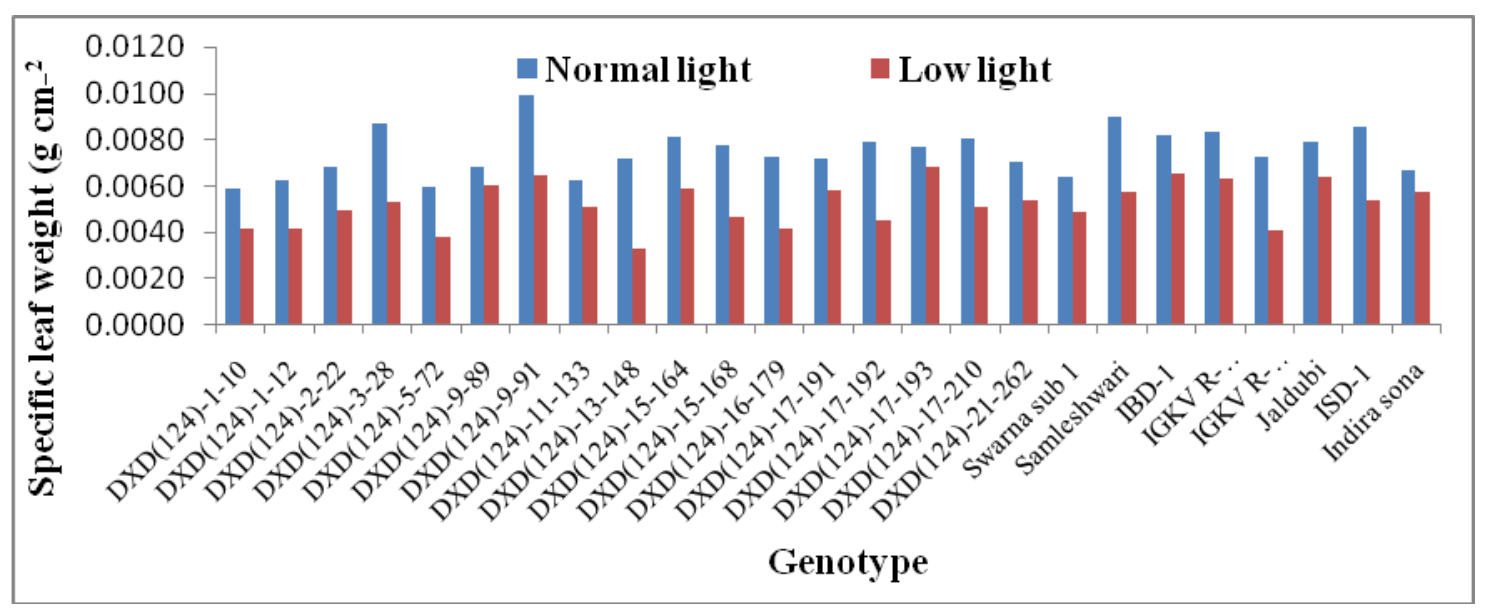

Fig.2 Effects of normal light (NL) and low light (LL) conditions on specific leaf weight of rice (Oryza sativa L.) genotypes 


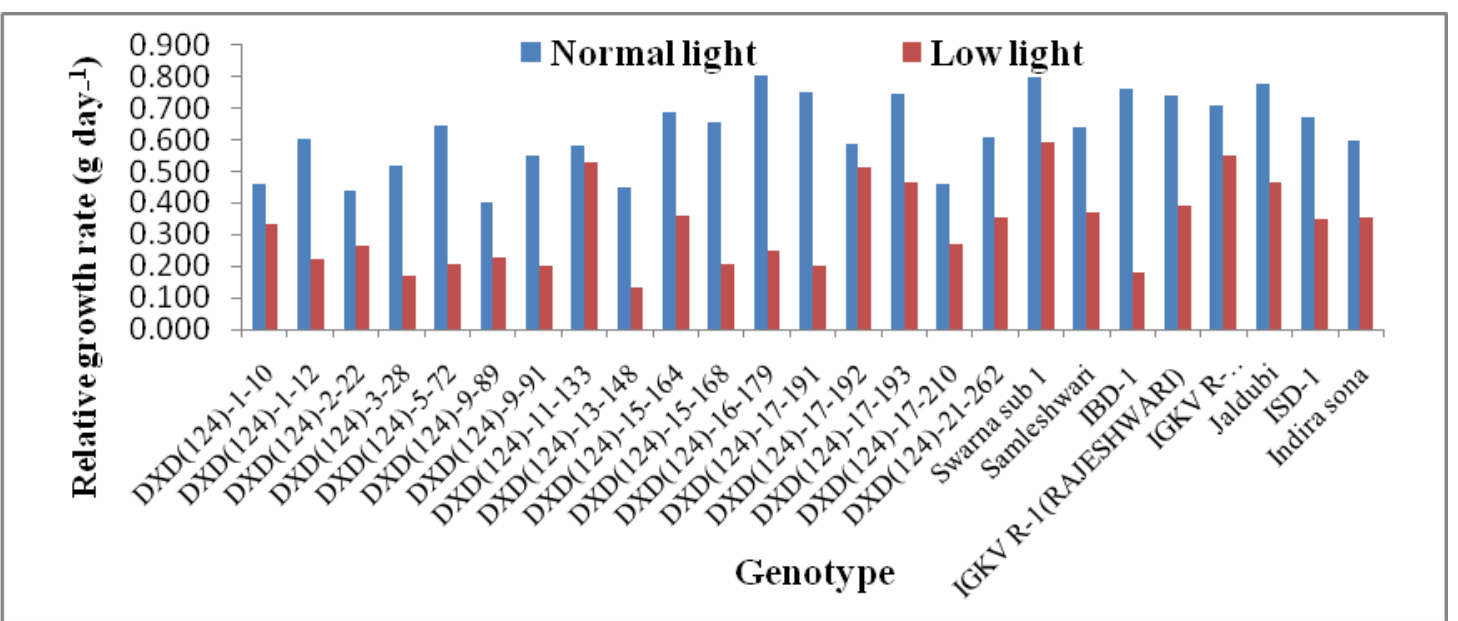

Fig.3 Effects of normal light (NL) and low light (LL) conditions on relative growth rate ( $\mathrm{g} \mathrm{day-}^{1}$ ) of rice (Oryza sativaL.) genotypes

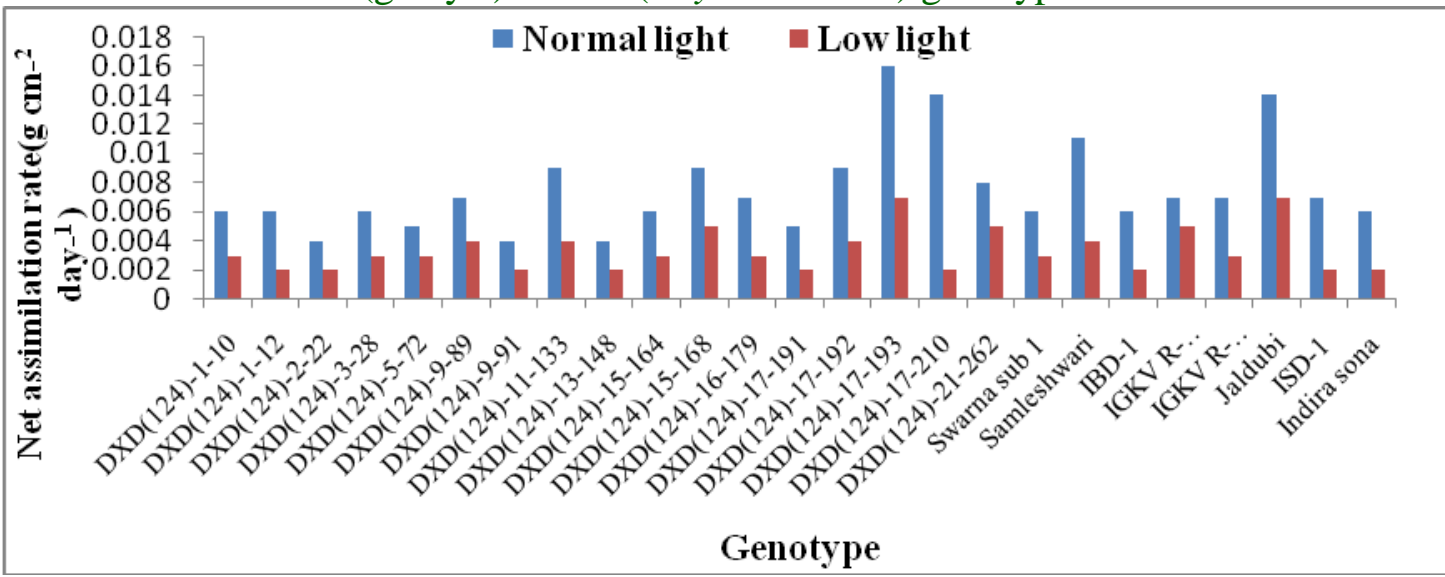

Fig.4 Effects of normal light (NL) and low light (LL) conditions on net assimilation rate $\left(\mathrm{g} \mathrm{cm}^{2}{ }^{2}\right.$ day- $\left.{ }^{1}\right)$ of rice (Oryza sativa $\mathrm{L}$.) genotypes

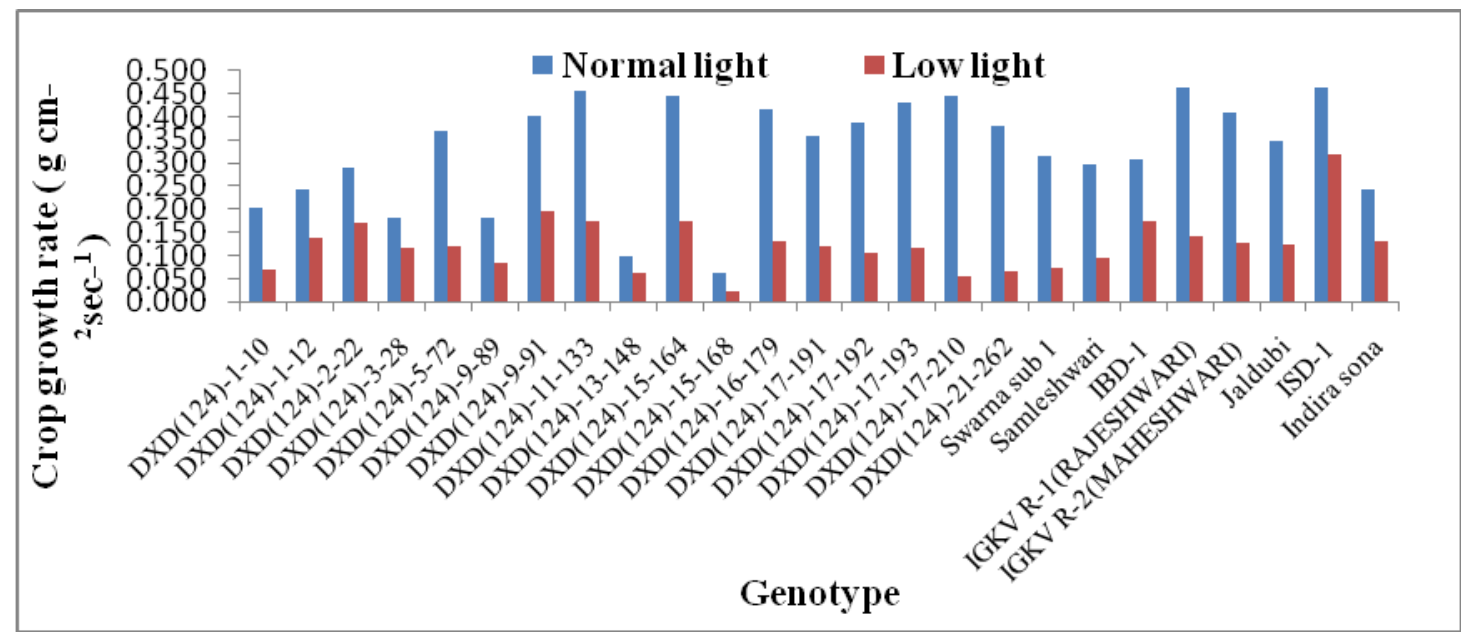

Fig.5 Effects of normal light (NL) and low light (LL) conditions on crop growth rate $\left(\mathrm{g} \mathrm{cm}^{-2} \mathrm{sec}^{-1}\right)$ of rice (Oryza sativa L.) genotypes 
Significant differences were noticed among the treatments in relation to crop growth rate (Table 2 and Fig. 5). Crop growth rate $\left(\mathrm{g} / \mathrm{m}^{2} /\right.$ day) at vegetative stage of crop was decreased under low light condition as contrast with normal light.

Overall mean decrease in crop growth rate was observed $35 \%$ under low light intensity. The variety Jaldubi exhibited maximum crop growth rate (CGR) under low light condition. It may be due to the low light condition, leaves are not able to receive sufficient light for the production of photosynthetic assimilates and they have a reduced ability to produce dry matter. Similar results were also reported by Sun et al., (2012).

\section{Acknowledgement}

This study was financially supported by KrishiVishwavidylaya DRS, IGKV, Raipur and Rajiv Gandhi National Fellowship (RGNF), New Delhi.

\section{References}

Ahmad, A.S., Iqbal, S., Ahmad, T., Khaliq, W., Nasim, Z., Husnain, A., Hussain, M., Zia-Ul-Haq and Hoogenboom, G. 2009. Seasonal growth, radiation interception, its conversion efficiency and biomass production of rice (Oryza ativaL.) Under diverse agro-environments in Pakistan. Pak. J. Bot.41: 1241- 1257.

Fageria, N.K. 2013.Mineral nutrition of rice. CRC press, Taylor and Francis Group. Boca Raton.
Janardhan, K. V., Murty, K S. 1980. Effect of low light during vegetative stage on photosynthesis and growth attributes in rice. Ind J Plant Physiol, 23(2): 156- 162 .

Koumotoa, T., Saitoa, N., Aokib, N., Iwasakic, T., Kawaia, S., Yokoia S., and Shimonoa, H. 2016. Effects of salt and low light intensity during the vegetative stage on susceptibility of rice to male sterility induced by chilling stress during the reproductive stage. Plant Production Science.

Li, T., Ohsugi, R., Yamagishi, T., Sasaki, H. 2005. Effects of weak light on rice starch accumulation and starch synthesis enzyme activities at grain filling stage. Chin J Rice Sci, 19(6): 545-550.

Sun, Y.Y., Sun, Y. J., Chen, L., Xu, H. and Ma, J. 2012. Effects of different sowing dates and lowlightstress at heading stage on the physiological characteristics and grain yield of hybrid rice. Ying Yong Sheng Tai ХиеВао, 23(10): 273-744.

Robakowski P., Montpied P. and Dreyer E. 2003. Plasticity of morphological and physiological traits in response to different levels of irradiance in seedling of silver fir (Abiesalba Mill.) Trees, (Berl.).7: 431-441.

Wang, L., Deng, F., Ren, W.J., and Yang, W.Y., 2013.Effects of Shading on Starch Pasting Characteristics of Indica Hybrid Rice (Oryza sativa L.). Plos one 8(7):68220.

\section{How to cite this article:}

Aradhana Dhruw and Arti Guhey. 2018. Effects of Low Light Stress on Growth Parameters of Rice (Oryza sativa L.) Genotypes. Int.J.Curr.Microbiol.App.Sci. 7(07): 472-478. doi: https://doi.org/10.20546/ijcmas.2018.707.057 